\title{
Beyond the hammer: The future of cardiothoracic surgery
}

Tom C. Nguyen, MD, ${ }^{a}$ and Isaac George, $\mathrm{MD}^{\mathrm{b}}$

See related commentary on page 678 .

I suppose it is tempting, if the only tool you have is a hammer, to treat everything as if it were a nail. -Abraham Maslow

"The law of the instrument," attributed to the American psychologist Abraham Maslow, applies now more than ever to the field of cardiac surgery. This theory presupposes that clinicians form inherent reliance on and bias toward familiar tools: surgeons primarily offer surgery to patients, whereas cardiologists mainly endorse percutaneous options. The recent innovation of minimally invasive valvular replacement techniques has generated increasing momentum toward managing complex disease with the least-invasive techniques. Patients, for their part, are increasingly seeking procedures that use the smallest incisions.

The need for the traditional cardiac surgeon to evolve to keep up with this paradigm shift is becoming more and more apparent. From our perspective as 2 recent graduates in surgery, we address key issues central to the growth of transcatheter aortic valve replacement (TAVR), minimally invasive surgery, the barriers involved in training new surgical graduates, and the future landscape for our field. We argue that training for graduates must be revised altogether to accommodate the explosion of new percutaneous technology, with a caveat that failure to do so will exclude surgeons from treating valve disease in the future.

The evolution of vascular surgery provides a valuable historical example of clinical adaptation as a method of specialty preservation. Vascular surgery originated as a branch of general surgery, treating a myriad of complex vascular cases that were not amenable to medical management. With the innovation of interventional radiology techniques, peripheral vascular disease patients who would have been surgical candidates previously began to be treated

\footnotetext{
From the Department of Cardiothoracic and Vascular Surgery, ${ }^{\mathrm{a}}$ University of Texas Medical School at Houston, Memorial Hermann Hospital-Heart and Vascular Institute, Houston, Tex; and Division of Cardiothoracic Surgery, ${ }^{\mathrm{b}}$ Department of Surgery, New York Presbyterian Hospital-College of Physicians and Surgeons of Columbia University, New York, NY.

Disclosures: Authors have nothing to disclose with regard to commercial support.

Received for publication Nov 29, 2014; accepted for publication Nov 30, 2014; available ahead of print Jan 23, 2015.

Address for reprints: Tom C. Nguyen, MD, University of Texas-Memorial Hermann, 6400 Fannin St, Suite 2850, Houston, TX 77030 (E-mail: tom.c. nguyen@gmail.com).

J Thorac Cardiovasc Surg 2015;149:675-7

$0022-5223 / \$ 36.00$

Copyright (c) 2015 by The American Association for Thoracic Surgery

http://dx.doi.org/10.1016/j.jtcvs.2014.11.091
}

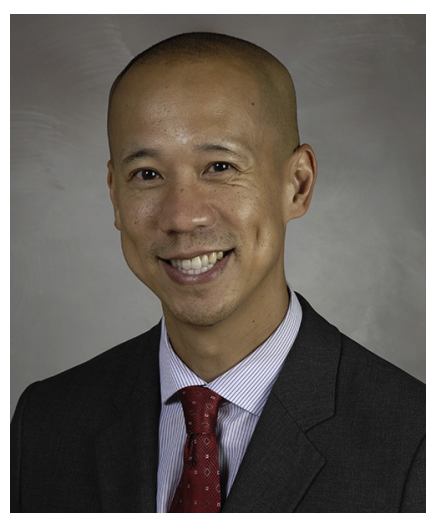

Tom C. Nguyen, MD

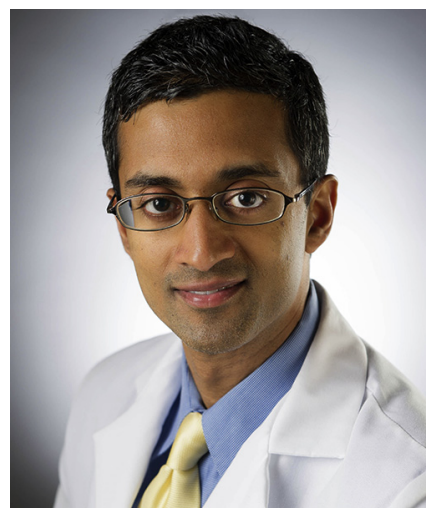

Isaac George, MD

instead with less-invasive endovascular procedures, experiencing benefits comparable to those from open surgery.

These developments might have had devastating consequences for vascular surgeons if it were not for their ability and willingness to learn new skills so that they could perform catheter-based interventions. Vascular surgeons proactively evolved to accommodate the 3 areas required for a transformation: wholesale clinical adoption, aggressive revision of training program paradigms, and subsequent investment in clinical innovation. In fact, vascular surgery was one of the first specialties to adopt a dual-system residency training program, which has flourished since its inception in 2005.

\section{CLINICAL ADOPTION}

In the same way, cardiac surgeons must be proactive about fully adopting and pioneering new techniques for the treatment of valvular heart disease. TAVR provides an ideal opportunity for cardiac surgeons to enter this niche, but this transition must be made soon. First performed in humans in 2002, by Alan Cribier, ${ }^{2}$ TAVR still relies on the skill sets of both surgeons and interventional cardiologists. In the PARTNER (Placement of Aortic 
Transcatheter Valves) trial, TAVR had a near 25\% survival benefit compared with medical therapy alone. ${ }^{3}$ Three-year follow-up studies have shown that these results are maintained, ${ }^{4}$ and TAVR is now recommended in select patient groups who are deemed inoperable or high-risk surgical candidates. ${ }^{5}$ The 2014 American College of Cardiology and American Heart Association guidelines for the management of valvular heart disease lists TAVR as a Class IIa recommendation in high-risk and inoperable patients who meet the criteria for aortic valve replacement. ${ }^{6}$

The disadvantages of TAVR in early trials, such as a higher incidence of paravalvular leak, stroke, and vascular complications, prompted discussion over the applicability of TAVR in intermediate- or low-risk populations. However, new data suggest that these concerns may be unfounded, with the use of smaller, lower-profile devices to reduce stroke and vascular complication, and special design features to improve performance and reduce paravalvular leak. Stroke rates and cardiovascular complications for TAVR are comparable to those for surgical AVR, with no difference in frequency of stroke, myocardial infarction, 30-day mortality, or 1-year mortality in current series. ${ }^{7}$ In addition, if TAVR becomes a viable treatment for intermediate-risk patients, this development will further deplete the patient pool. The cardiac surgeon whose only tool is to operate will be excluded from caring for these patients in the future.

Surgeons who adopt transcatheter therapy and minimally invasive techniques are in a unique position because of their ability to provide true equipoise in management decisions. By adding more tools to their treatment toolbox, they can evolve past the "law of the instrument." Although cardiologists can offer only TAVR, the surgeon who is trained in endovascular, minimally invasive surgery, and conventional surgery (ie, sternotomy) can more objectively provide a range of therapeutic options. This approach can strengthen the foundation of a collaborative heart team-a joint effort among cardiologists and surgeons that provides the patient with the most comprehensive care possible.

As indications for TAVR risks decrease, obstacles to entry into the area of transcatheter valve therapy may become insurmountable for traditional cardiac surgeons. Currently, the Centers for Medicare \& Medicaid Services require the presence of both cardiologists and surgeons for TAVR reimbursement. If this requirement were ever discontinued, which is not inconceivable given the rapid evolution of TAVR toward more transfemoral approaches, then surgeons who are not intimately involved with all aspects of transcatheter procedures may see a decline in case volume. Just as vascular surgeons have evolved to incorporate nonsurgical techniques in the care of their patients, cardiac surgeons must recognize that a portion of their business will need to be nonsurgical to ensure the survival of their specialty. This news may be disappointing to those who entered the field primarily for their love of operating, but it is the stark reality as patients seek less-invasive options. In addition, collaboration between surgery and cardiology is going to be essential to ensure the best possible outcome in light of the explosion in transcatheter technology.

\section{TRAINING}

The question then arises: How can cardiac surgeons best incorporate catheter-based interventions into their practice? Unfortunately, this transition is not as easy as it may seem. For instance, many surgeons currently perform the "surgical" component only of transapical and transaortic TAVRs (ie, femoral artery cut-down, left thoracotomy, hemisternotomy, holding the sheath, inflating the balloon during deployment, etc), whereas the cardiologist actually leads the procedure. To be truly proficient, cardiac surgeons must be able to lead the procedure and independently and autonomously perform the entire procedure from beginning to end, including all the endovascular aspects. To do this, surgeons will have to change their mindset regarding their role in the procedure and be willing to learn the technology. This adaptation requires acquisition of not only technical expertise, but also knowledge of appropriate patient selection, wire types, potential complications, and postoperative management. For surgeons to successfully add a new tool to their toolbox, they will have to know how to fully utilize it.

As added incentive to learn endovascular procedures, a rapidly growing shift is occurring, from transapical and transaortic TAVR, to the transfemoral approach, which means even less surgical involvement. In clinical comparisons between transfemoral and transapical TAVR, the transfemoral approach consistently results in a lower 30-day mortality rate compared with the transapical method, despite risk adjustment. ${ }^{8}$ Surgeons can no longer be content to just perform the femoral artery cut-down or hold the sheath for TAVR.

Once surgeons change their mindset regarding their role in the endovascular treatment of valvular heart disease, how do they acquire the skills they need to become proficient? The first step is to master traditional surgical techniques to treat complex structural heart disease. Next comes the understanding that procedures such as TAVR are not necessarily simple; they require a thorough understanding of the nuances of every aspect of the procedure. Third, surgeons need to be comfortable with managing possible endovascular complications, including the use of stent grafts for dissections and ruptures. Finally, they will have to learn the appropriate preoperative, intraoperative, and postoperative management of these patients, which differs from that for standard surgical care.

Learning these aspects of TAVR cannot be performed over a period of a few months with casual observation; specialized and dedicated training are required to fully become an endovascular cardiac surgeon. For residents, this requirement means pursuing a dedicated Structural Heart Fellowship 
over a period of 6 months to 1 year, in contrast to a mentorship-type setting with older surgeons who are still in the process of integrating catheter-based procedures into their own practices. Specific training over a minimum of 6 months will ensure adequate time and supervision to independently perform the procedures. Percutaneous skills apply to not only TAVR, but also all structural heart interventions (percutaneous mitral valve repair and/or replacement, percutaneous tricuspid valve repair and/or replacement, pulmonic valve replacement, etc). It is incumbent on those in the field of cardiac surgery to foster these specialty-training programs and encourage their graduates to enroll.

Furthermore, graduates may be tempted to seek out the highest-paying or highest-status specialties, or to perform the most difficult procedures in training while avoiding percutaneous procedures. Although we understand that not doing open surgery has an opportunity cost, we strongly believe that acquisition of these percutaneous skills in training will pay large dividends in the future. This outcome was truly the case for the coauthors of this editorial; we spent 1 year in structural heart endovascular programs at Emory University and Columbia University after our training. The Thoracic Surgery Residency Review Committee and American Board of Thoracic Surgery recognize the importance of wire skills and now require graduates to perform 20 interventional procedures, but this number is not nearly enough.

\section{INNOVATION}

Clearly, TAVR not only is here to stay, but also reflects a growing trend in the treatment of all structural heart disease. Numerous devices for transcatheter mitral valve repair are being investigated in phase I and II clinical trials, some of which have the potential to replace surgical techniques such as annuloplasty and chordal reconstruction. ${ }^{9}$ In addition, the past decade has seen a growth in transcatheter approaches to treating pulmonary valve disorders, both as primary treatments and as postsurgical interventions to avoid repeat open-heart surgery. ${ }^{10,11}$

Cardiac surgeons must be in the frontline, innovating, adopting, and leading the development of newer technologies. Surgeons have an intimate knowledge of the heart that is unmatched by any specialty; translation of this knowledge into structural heart valve technology is logical and needed. Cardiologists have been intimately involved in the process of developing sophisticated endovascular devices, and have therefore become the leaders in catheter-based techniques. If surgeons are able to expand their mindset in this area, they can contribute valuable knowledge and unique expertise gained from decades of treating structural heart disease. This change will only lead to newer and better technology that draws on the strengths that both cardiology and surgery have to offer.

\section{CONCLUSIONS}

Minimally invasive, catheter-based therapies for valvular heart disease have disrupted the standard of surgical care for valve disease, but these techniques need not be relegated to the domain of cardiology only. In fact, to provide the best comprehensive care for patients, a collaborative heart team that includes cardiologists and surgeons who are all equally proficient in endovascular techniques is essential. Surgeons who can operate independently of cardiologists will only accelerate the growth of new technologies, offering their valuable input in how best to approach the conditions they have already been treating for decades. To accomplish this change, the following are required: a significant time commitment in training, acceptance of the technology with a generational shift in mindset, and a desire to innovate and provide leadership. Although residents may be able to learn certain aspects of the procedure during their training programs, true proficiency will require a dedicated fellowship.

The cardiac surgeons of the past must transform into the surgeons of the future. Only by understanding the law of the instrument will surgeons begin to see valvular heart disease as more than a nail waiting to be hammered.

T.N. is indebted to Dr Robert Guyton and Dr Vinod Thourani for their unselfish mentorship, clinical mastery, and steadfast leadership. I.G. has been honored to train with a true surgical pioneer in the field of transcatheter valve and interventional cardiology, Dr Mathew R. Williams.

\section{References}

1. Jones WS, Dolor RJ, Hasselblad V, Vemulapalli S, Subherwal S, Schmit K, et al. Comparative effectiveness of endovascular and surgical revascularization for patients with peripheral artery disease and critical limb ischemia: systematic review of revascularization in critical limb ischemia. Am Heart J. 2014;167:489-98.

2. Cribier A, Eltchaninoff H, Bash A, Borenstein N, Tron C, Bauer F, et al. Percutaneous transcatheter implantation of an aortic valve prosthesis for calcific aortic stenosis: first human case description. Circulation. 2002;106:3006-8.

3. Leon MB, Smith CR, Mack M, Miller DC, Moses JW, Svensson LG, et al Transcatheter aortic-valve implantation for aortic stenosis in patients who cannot undergo surgery. N Engl J Med. 2010;363:1597-607.

4. Kapadia SR, Tuzcu EM, Makkar RR, Svensson LG, Agarwal S, Kodali S, et al. Long-term outcomes of inoperable patients with aortic stenosis randomly assigned to transcatheter aortic valve replacement or standard therapy. Circulation. 2014;130:1483-92.

5. Webb J, Cribier A. Percutaneous transarterial aortic valve implantation: what do we know? Eur Heart J. 2011;32:140-7.

6. Nishimura RA, Otto CM, Bonow RO, Carabello BA, Erwin JP III, Guyton RA, et al. 2014 AHA/ACC Guideline for the management of patients with valvular heart disease: Executive summary: A Report of the American College of Cardiology/American Heart Association Task Force on practice guidelines. Circulation. 2014;129:2440-92

7. Nagaraja V, Raval J, Eslick GD, Ong AT. Transcatheter versus surgical aortic valve replacement: a systematic review and meta-analysis of randomised and non-randomised trials. Open Heart. 2014;1:e000013.

8. Li X, Kong M, Jiang D, Dong A. Comparison 30-day clinical complications between transfemoral versus transapical aortic valve replacement for aortic stenosis: a meta-analysis review. J Cardiothorac Surg. 2013;8:168.

9. Piazza N, Asgar A, Ibrahim R, Bonan R. Transcatheter mitral and pulmonary valve therapy. J Am Coll Cardiol. 2009;53:1837-51.

10. Lurz P, Bonhoeffer P, Taylor AM. Percutaneous pulmonary valve implantation: an update. Expert Rev Cardiovasc Ther. 2009;7:823-33.

11. Frigiola A, Nordmeyer J, Bonhoeffer P. Percutaneous pulmonary valve replacement. Coron Artery Dis. 2009;20:189-91. 